\title{
Invasive breast carcinoma with MRI kinetic curve type I
}

\section{Christa Levina Daniswara \\ Rumuat Semuel Wullul Manangka}

Department of Radiology, Mayapada Hospital Lebak Bulus, South Jakarta, Indonesia
Cite this article:

Daniswara, CL, Manangka RSW. Invasive breast carcinoma with MRI kinetic curve type I.

Neurologico Spinale Medico Chirurgico.

2020.3(3): 122-129.

DOI: 10.36444/nsmc.v3i3.126

\section{Corresponding author:}

Christa Levina Daniswara

Department of Radiology,

Mayapada Hospital Lebak Bulus,

South Jakarta, Indonesia

Orcid ID: 0000-0003-443।-9745

christalevina09@gmail.com

\begin{abstract}
Breast cancer is the most common cancer in women and is the most significant cause of morbidity and mortality in women cancer patients. Increased awareness of people followed by periodic screening, appropriate supporting examinations, and imaging modalities according to the patient's condition could improve prognosis.

We report a case report of a 55-year-old woman with a complaint of a lump in the right breast. Ultrasound examination and mammography showed a single solid lesion in the superior quadrant of the right breast corresponding to Breast Imaging-Reporting and Data System (BI-RADS) 4c. Magnetic Resonance Imaging (MRI) examination revealed a single lesion with enhancement curve type 1 according to bi-rads $4 \mathrm{~b}$. Results of the biopsy were grade 2 breast carcinoma without any lymph node metastases.

The use of a single modality cannot be used as a benchmark in determining the best diagnosis and therapy. The kinetic feature/enhancement curve of Dynamic ContrastEnhanced MRI (DCE-MRI) cannot be used as the main guide for determining the diagnosis. The morphology of the lesion either from ultrasound, mammography, or MRI should also be considered in determining the diagnosis and treatment plan.
\end{abstract}

Keywords: Breast cancer, Enhancement curve, Imaging modalities, Kinetic curve, Breast MRI

\section{Introduction}

Breast cancer is the most common cancer in women and the most significant cause of morbidity and mortality in the world. ${ }^{1}$ In 2008 , the number of deaths reached 8 million and is expected to reach 11 million by $2030 .^{2}$ In 2012 , as many as 1.67 million new cases were found worldwide, about $25 \%$ of all cancer cases. In 2017, approximately 252,710 new cases were found, of which around 24\% were found in the Asia Pacific region, with the highest cases in China, Japan, and Indonesia. ${ }^{2}$ Increasing population awareness, especially those with high risks will result in earlier examination and diagnostic procedures performed which could improve prognosis by early diagnosis. Mammography has been shown to be able to detect breast cancer at an early stage, especially in women over 40 years. In cases where mammography is less sensitive (women under 40 years of age) ultrasound or Magnetic Resonance Imaging (MRI) may be considered. ${ }^{1} \mathrm{MRI}$ is an important imaging modality in breast imaging, as recommended by the American and European guidelines for breast cancer. Breast 
MRI is the most sensitive modality for detecting malignancy, but it has low specificity. ${ }^{3}$

The sensitivity of breast MRI is reported to be $90 \%$, while the specificity is still low, around $72 \%$ which makes the determination of benign or malignant lesions still difficult to establish. ${ }^{4}$ Breast MRI has a special feature to evaluate the morphology of the lesion as well as to analyze the permeability of the vascularization structure supplying the lesion found from kinetic curve Dynamic Contrast-Enhancement MRI (DCE-MRI). Theoretically, benign lesion will show a progressive enhancement pattern with a continuous increase in signal intensity (curve type I) and malignant lesion will produce a type III kinetic curve pattern. ${ }^{3}$ In this case report, the morphology of the lesion from ultrasound and mammography examinations showed the characteristics of malignancy, yet from the MRI kinetic curve examinations showed a type I kinetic curve MRI. Therefore, this case is an interesting one to be analyzed further.

\section{Case report}

A 55-year-old woman came to the outpatient clinic with a chief complaint of a lump in the right breast felt since one month ago. The lump was hard, there was not a sudden increase in lump size. The patient has two children and has not yet experienced menopause. A history of discharge from the nipple and a history of breast cancer in the family were denied. The breast lump is invisible and the size and shape of both breasts are still symmetrical. Through palpation, a single lump in the superior quadrant of the right breast with a diameter of $2 \mathrm{~cm}$, hard, immobile, solid lump was found. No nipple discharge/retraction was found in the skin of the lump area.

The patient underwent an ultrasound, mammography, and breast MRI examination. Both ultrasound and mammography showed a single solid lesion in the superior quadrant of the right breast according to bi-rads 4c, without the presence of enlarged lymph nodes. MRI revealed a single solid lesion with curve enhancement type 1 according to bi-rads $4 \mathrm{~b}$, with malignancy as the main differential diagnosis, followed by focal mastitis as the second possibility.

The patient eventually underwent breast-conserving surgery (BCS) and sentinel node biopsy with the frozen-section method during intraoperative. Biopsy results showed invasive carcinoma grade 2 without carcinoma metastases in the sentinel lymph node. Furthermore, after surgery, the patient underwent a series of chemotherapy and radiotherapy.

\section{Discussion}

In general, the use of ultrasound is more recommended for young women due to the fibroglandular tissue component of the mammary. Since the sensitivity of mammography lower in women with dense breasts, the use of ultrasound is more favored in dense breast cases. ${ }^{5}$ Malignant lesions will generally present a hypoechoic lesion with indistinct borders. Characteristics of malignant lesions are usually hypoechoic lesions with spiculated borders, posterior acoustic shadowing, and microcalcification. ${ }^{6}$ Predictive values for benign lesions according to Hong et $a ., l$ include circumscribed borders $(90 \%)$, parallel orientation $(78 \%)$, and oval shape $(78 \%)$, whereas those for malignant lesions include irregular shapes (62\%), spiculated margins $(86 \%)$ and non-parallel orientation $(69 \%) .{ }^{7}$ Increased vascularity through color Doppler can also help determine signs of malignancy. ${ }^{6}$ Since 2003, BI-RADS has been established by the American College of Radiology (ACR) as a guideline to standardize interpretations, report, and improve communication between radiologists and clinicians.

In this case, it can be seen from the ultrasound image showing a heterogeneous breast composition, a single solid lesion with irregular shapes, indistinct borders, non-parallel orientation, and heterogeneous echo pattern. There were no calcifications, architectural changes, skin thickening, and skin retraction. From these characteristics, this lesion was suspicious of malignancy categorized as bi-rads 4 (Figure 1).

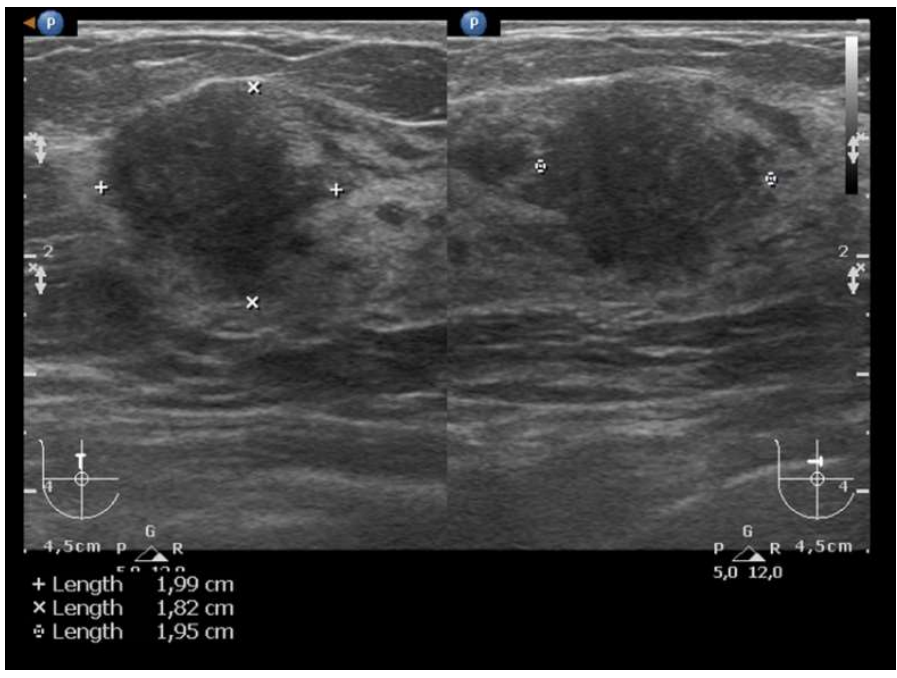

Figure 1. A single solid lesion with irregular shape and indistinct margin seen on ultrasound examination

In theory, Bi-rads 4 can be a benign lesion, although unlikely. Bi-rads $4 \mathrm{c}$ is established when new irregular and indistinct lesions are found or if a new fine linear calcification group is found. ${ }^{8}$ In accordance 
with this case, category $4 \mathrm{C}$ was assigned to moderate suspected cases, but did not show classic symptoms of malignancy. ${ }^{7}$ Lesion in this case only showed the irregular shape and non-circumscribed margin so that the Bi-rads category 5 with the possibility of malignancy of $95 \%$ is still not considered. Bi-rads 4 with a probability of malignancy varies from $3-94 \%$, with category $4 \mathrm{c}$ the probability of malignancy is $50-94 \%$. $^{7}$ The lesion characterized by irregular shape and non-parallel orientation showed positive predictive values of $62 \%$ and $69 \%$ so that this lesion fits into the category of $4 \mathrm{c}$ bi-rads.

Mammography is generally used in women over the age of 40 years. This is due to the fat component that increases with age. However, the use of mammography is recommended starting at the age of 30 years in women with several risk factors such as BRCA1 or BRCA 2 mutations, family history with breast cancer, or a history of radiation use. The use of mammography is not recommended before 25 years of age. ${ }^{5}$ Mammography is not used to rule out a diagnosis of breast cancer, but is used for detection and not for diagnosis. Mammography can be a diagnostic tool in some benign lesions such as lipoma or hamartoma. The obvious characteristic of a malignant lesion such as a spiculated mass is a highly suggestive sign but not a diagnosis of cancer. Therefore, either mass, calcification, architectural distortion, or a combination of these features should be evaluated with other modalities or a biopsy should be performed. The limitation of using mammography is dense breast condition so that the sensitivity decreases. ${ }^{8}$

From mammography examination, the breast composition is heterogeneously dense, solid lesions were found with irregular shape, indistinct, and spiculated margin in the superior quadrant (Figure 2). There was no asymmetry, architectural distortion, and calcification.

In theory, Bi-rads 4 can be a benign lesion, although unlikely. Bi-rads $4 \mathrm{c}$ is established when new irregular and indistinct lesions are found or if a new fine linear calcification group is found. ${ }^{8}$ Lesion with irregular shape and spiculated margins make malignancy as the main suspicion. Bi-rads 5 is established when there is a combination of irregular spiculated mass with fine linear calcification or irregular spiculated mass with associated pleomorphic calcification. ${ }^{8}$ However, no calcification was found to make bi-rads $4 \mathrm{c}$ more suitable than birads 5 .

Mammography is very sensitive in detecting microcalcifications, although it is not the main clue in determining benign or malignant lesions. ${ }^{9}$ The abnormalities identified on mammography are not specific for malignant lesions and their morphological features may reveal various degrees of suspicion for malignant lesions. A biopsy is still needed to make a definite diagnosis. $^{8,9}$

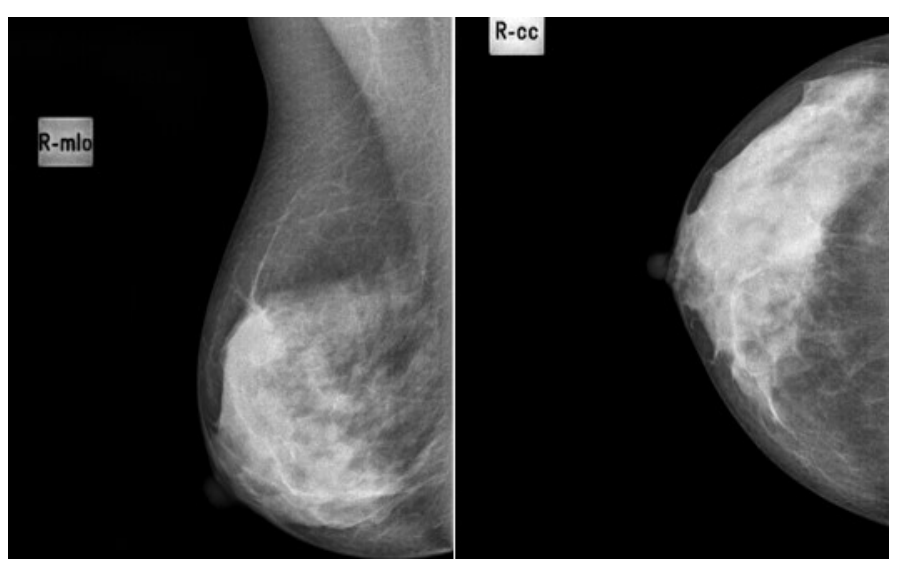

Figure 2. A single solid lesion with spiculated margin at the superior quadrant of right breast seen on mammography

The use of MRI is increasing along with its use as a screening, diagnostic evaluation, and surveillance. Mammography is used more as a screening method in a broad population, whereas MRI is used in cases of patients with symptoms suggestive of breast cancer. ${ }^{10}$ Several studies comparing ultrasound, mammography, and MRI as screening methods, found that MRI is superior especially in high-risk patients. Screening method using ultrasound may have a role as supplemental screening for high-risk patients with contraindication to MRI. ${ }^{5}$

Indications for the use of breast MRI in general include staging methods, screening of high-risk patients, and evaluation of chemoadjuvant therapy. ${ }^{3}$ According to the American Cancer Society (ACS) 2007, MRI is used to screen asymptomatic women with a high risk of breast cancer defined as: 1) known carriers of BRCA gene mutations; 2) first-degree relatives of known carriers of the BRCA gene mutation; or 3 ) women with $>20 \%$ lifetime risk of breast cancer. ${ }^{10}$ Based on the journal American College of Radiology, the use of MRI is used in cases of women with breast cancer risk starting at the age of 30 years, a history of chest area radiation, as well as patients diagnosed with breast cancer with normal contralateral breast. MRI breast is not intended to replace the use of mammography in detecting breast cancer. In the case of DCIS, it can be detected only through mammography but not through MRI. The use of MRI and mammography can be used simultaneously or alternately within 6 months as a screening method. ${ }^{5}$ Compared to mammography, MRI has better specificity so that it can lead to a higher risk of false positives, more expensive, and requires intravenous contrast administration. ${ }^{10} \mathrm{~A}$ false positive result may cause patient anxiety, unnecessary biopsies, and even over-treatment. ${ }^{11}$ 
The principle of using MRI today is the multiparametric technique with T2-weighted and diffusion-weighted imaging (DWI) which is routinely used, as well as dynamic T1-weighted contrastenhanced sequences. ${ }^{12}$ DWI has been shown to be less helpful in distinguishing malignant or benign lesions. The main roles of DWI in MRI include predicting treatment outcomes, for tumor staging, and identifying lymph node involvement. DWI is measured by Apparent Diffusion Coefficient (ADC) values as indicated by the diffusion of water through the tissues. ADC value in malignant lesions was lower compared to benign lesions. Benign lesions have higher ADC values due to normal cellularity and the absence of restriction on water movement. ${ }^{13}$

Dynamic contrast-enhancement MRI (DCE-MRI) analysis measures the permeability of the vasculature supplying the lesion. In the case of leaky vessels, accumulated contrast will be removed and eliminated from the lesion. In the case of less-permeable blood vessels, the contrast will gradually pass through the blood vessels, so that the enlargement of the lesion will continue to increase. ${ }^{12}$

Standard breast MRI techniques include DCE with a T1weighted sequence performed before and after gadolinium-based contrast injection. For clinical purposes, benign and malignant lesions are differentiated by morphological evaluation and kinetic curve assessment. ${ }^{14}$ Morphological identification with high spatial resolution from MRI by assessing the morphological margin or internal architecture of the lesion. ${ }^{11}$ The kinetic curve shows the signal enhancement in relation to the time after contrast injection. ${ }^{14}$ The combination of kinetic and morphological features are required for an accurate diagnosis. ${ }^{11}$ Recently, the combination of morphological characteristics and kinetic curve sensitivity was reported to be $97 \%$ and their specificity up to $76.5 \%$. $^{9}$

The kinetic curve from DCE is able to describe the hemodynamic features of a specific lesion. Kinetic curves can be classified into 3 categories. It is called early enhancement if there is enhancement within 2 minutes after the injection of contrast. Early enhancement can be further divided into slow, medium, and rapid, wherein rapid enhancement (within 90 seconds) strongly represents malignancy. The increase in signal intensity 2 minutes after contrast injection is known as the delayed phase. The delayed phase is divided into 3 types, including type 1 "persistent", type 2 "plateau" and type 3 "washout". Persistent (type 1) shows a progressive enhancement pattern with a continuous increase in signal intensity. Persistent curves usually represent benign lesions. Plateau (type 2) shows peak enhancement after contrast injection, followed by flattening during the delayed phase.
Plateau curves can show both in benign and malignant lesions. ${ }^{15}$
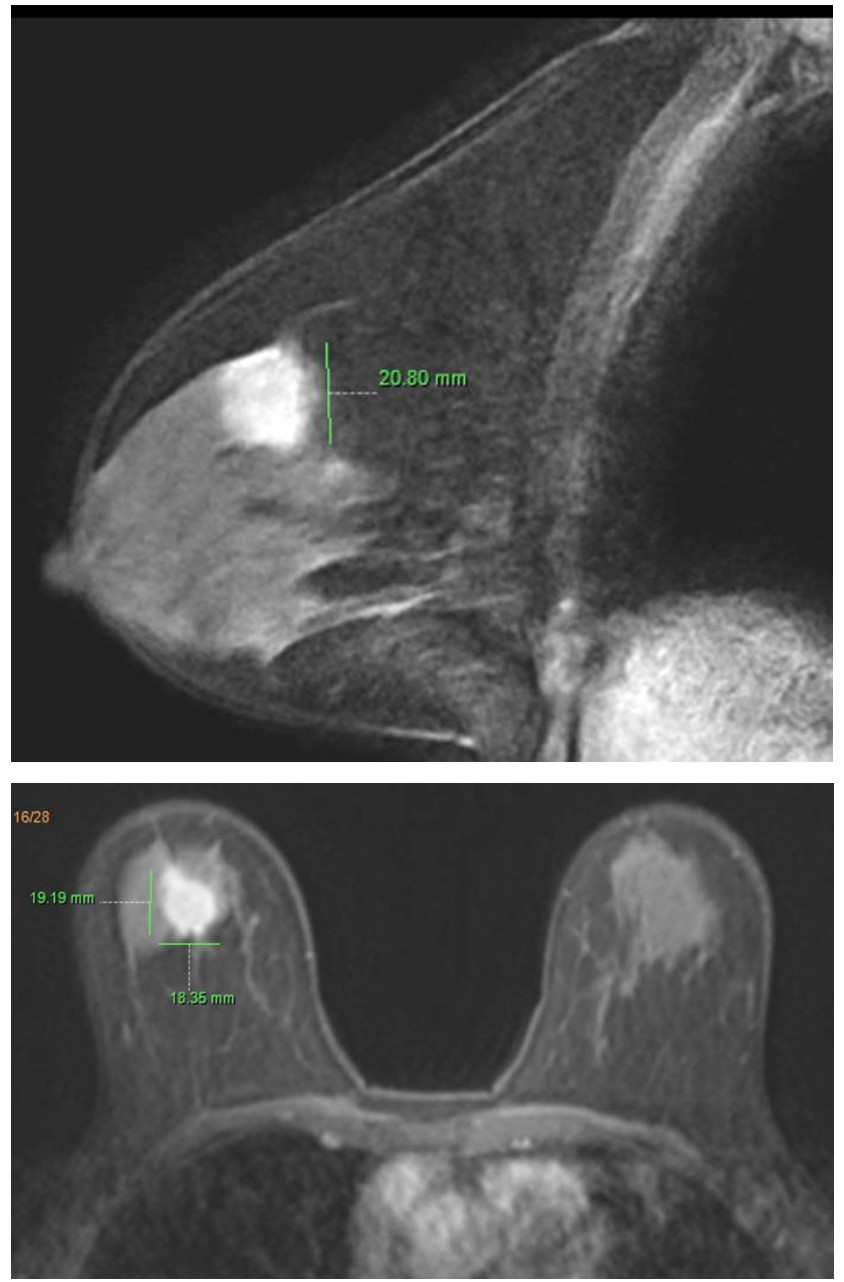

Figure 3. T1-weighted contrast-enhanced sequences showed enhancement after contrast administration

Another study argues that the plateau curve is a feature of malignant lesions. ${ }^{11}$ Washout (type 3 ) showed an increase in signal enhancement followed by a decrease in signal intensity after 2 minutes of contrast injection. Initial increased signal enhancement indicates tumor angiogenesis. ${ }^{15}$ In other words, malignancy is characterized by rapid improvement in the early phase and late washout, while slow early onset and late persistent washout show the features of benign lesions. ${ }^{14}$ Bluemke et al., indicated that both plateau and washout were malignant lesions. ${ }^{11}$ Kinetic curve helps to diagnose and determine the treatment of lesions that are morphologically benign. In cases where the lesion is suspected to be malignant, a biopsy should be performed regardless of the shape of the kinetic curve. ${ }^{15}$

American College of Radiology (ACR) recommends that the morphology of the lesion should be reported using the standardized BIRADS. Characteristics of malignant lesions seen on DCE-MRI include 
larger size, irregular or spiculated margin, washout type curve (type III), or heterogeneous enhancement. ${ }^{16}$ Morphologically, this case showed a suspicious shape and borders in malignant features, so that it was suitable to be categorized as BI-RADS 4 and biopsy should be performed (Figure 3).

In this case, the kinetic curve of the breast MRI showed a persistent increase in the intensity of the enhancement (type 1) indicating the feature of the benign lesion (Figure 4).

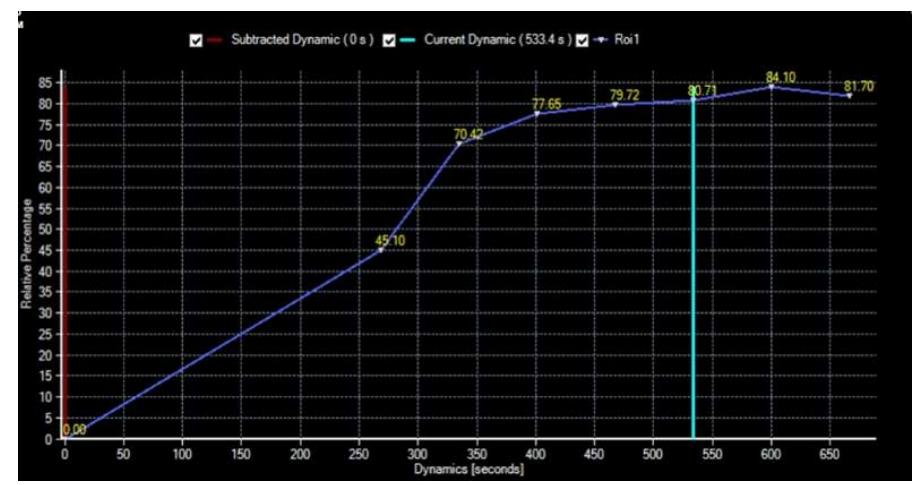

Figure 4. The kinetic curve of breast MRI showed a persistent increase in the intensity of the enhancement (type 1)

However, morphologically both ultrasound and mammography also support the diagnosis in the feature of malignant lesions. Although in this case the kinetic curve indicated the possibility of benign lesions, in the general margin and shape analysis (morphologically) are the most predictive features in the interpretation of MRI images. ${ }^{9}$ MRI examination showed indistinct lesions and mammography also showed spiculated margins. Spiculated margins are warning signs for carcinoma, with a positive predictive value for malignant lesions reaching $91 \% .{ }^{9}$ The kinetic curve pattern itself cannot be relied upon as a diagnostic determinant, seen in the case of DCIS only about $70 \%$ of cases showed enhancement in the initial phase with variable delayed phase enhancement. ${ }^{11}$ DCE feature on MRI is more accurate than mammography or ultrasound in identifying the extent of disease but has limitations in distinguishing between malignant and benign lesions. ${ }^{13}$ The study conducted by Ghazala et al., showed that MRI was the most sensitive modality for detecting malignant lesions but had limited specificity due to overlapping characteristics between benign and malignant. ${ }^{9}$

Generally, radiology modalities used to detect breast cancer are ultrasonography, mammography, and breast MRI. Each modalities have their own advantages and limitations as explained in this article. Several considerations in selecting the modalities to detect breast cacer such as the patient's age, breast cancer risk factor, breast density and the result from previous examinations or screening. ${ }^{5}$ In daily practice, selecting the right modalities for each patient could be the solution to detect breast cancer as early and as accurate as possible.

Table 1. Sensitivity and specificity each modalities ${ }^{4,18}$

\begin{tabular}{cccc} 
& Breast Ultrasound & Mammography & Breast MRI \\
\hline Sensitivity & $82 \%$ & $49 \%$ & $90 \%$ \\
Specificity & $84 \%$ & $89 \%$ & $72 \%$
\end{tabular}

\section{Conclusion}

Mixed imaging modalities can provide significant information for the diagnosis, staging, and treatment planning. ${ }^{17}$ Therefore, the use of a single modality can't be used as a benchmark in determining the best diagnosis and therapy for patients. Knowledge of the strengths and weaknesses of each imaging modality is also important in determining the diagnosis. MRI has a DCE-MRI feature as one of its advantages over the US and mammography. However, this kinetic curve alone cannot be used as a confirmation for diagnosis. The morphology of the lesion also plays an important role in reports according to BI-RADS standards, determining the diagnosis, and of course the best treatment planning for the patient.

\section{References}

1. Elwakeel A, Omar S, Bayoumy A. Role of magnetic resonance imaging in differentiation of benign and malignant breast lesions. Menoufia Medical Journal. 2018;31:502-507. DOI: 10.4103/mmj.mmj_623_16

2. Momenimovahed Z, Salehiniya H. Epidemiological characteristics of and risk factors for breast cancer in the world. Breast Cancer (Dove Med Press). 2019;11:151-164. DOI: 10.2147/BCTT.S176070

3. Millet I, Pages E, Hoa D, et al. Pearls and pitfalls in breast MRI. The British Journal of Radiology. 2012;85(1011):197-207. DOI: 10.1259/bjr/47213729

4. Radhakrishna S, Agarwal S, Parikh P, et al. Role of magnetic resonance imaging in breast cancer management. South Asian Journal of Cancer. 2018;7(2):69-71. DOI: 10.4103/sajc.sajc_104_18

5. Lee C, Dershaw D, Kopans D, et al. Breast cancer screening with imaging: recommendations from the Society of Breast Imaging and the ACR on the use of mammography, breast MRI, breast ultrasound, and other technologies for the detection of clinically occult breast cancer. Journal of the American College of Radiology. 2010;7(1):18-27. DOI: 10.1016/j.jacr.2009.09.022 
6. Gokhale S. Ultrasound characterization of breast masses. Indian Journal of Radiology and Imaging. 2009;19(3):242-247. DOI: 10.4103/0971-3026.54878

7. Raza S, Goldkamp A, Chikarmane S, et al. US of breast masses categorized as BI-RADS 3, 4, and 5: pictorial review of factors influencing clinical management. Radiographics. 2010;30(5):11991213. DOI: $10.1148 /$ rg.305095144

8. Thulkar S, Hari S. Present role of mammography/digital mammography in breast cancer management. PET Clinics. 2009;4(3):213-225. DOI: 10.1016/j.cpet.2009.09.006

9. Ghazala S, Elgohary M, Zidan D, et al. Characterization of suspicious breast lesions with dynamic contrast enhanced MRI in comparison to conventional mammography and ultrasonography. J Cancer Prev Curr Res. 2016;4(3):00121. DOI: 10.15406/jcpcr.2016.04.00121

10. Wernli K, DeMartini W, Ichikawa L, et al. Patterns of breast magnetic resonance imaging use in community practice. JAMA Internal Medicine. 2014;174(1):125-132. DOI: 10.1001/jamainternmed.2013.11963

11. Agrawal G, Su M, Nalcioglu O, et al. Significance of breast lesion descriptors in the ACR BI-RADS MRI lexicon. Cancer. 2009;115(7):1363-1380. DOI: $10.1002 /$ cncr.24156

12. Mann R, Cho N, Moy L. Breast MRI: state of the art. Radiology. 2019;292(3):520-536. DOI: 10.1148/radiol.2019182947
13. Youssef $\mathrm{M}$, Elahwal $\mathrm{H}$, Alwageeh $\mathrm{M}$, et al. Role of MRI in differentiating benign from malignant breast lesions using dynamic contrast enhanced MRI and diffusion weighted MRI. Alexandria Journal of Medicine. 2018;54(1):1-9. DOI: 10.1016/j.ajme.2016.12.008

14. Abe H, Mori N, Tsuchiya K, et al. Kinetic analysis of benign and malignant breast lesions with ultrafast dynamic contrast-enhanced MRI: comparison with standard kinetic assessment. American Journal of Roentgenology. 2016;207(5):1159-1166. DOI: 10.2214/AJR.15.15957

15. Cheng $\mathrm{L}, \mathrm{Li} \mathrm{X}$. Breast magnetic resonance imaging: kinetic curve assesment. Gland Surgery. 2013;2(1):50-53. DOI: 10.3978/j.issn.2227684X.2013.02.04

16. Leithner D, Wengert G, Helbich T, et al. Clinical role of breast MRI now and going forward. Clinical Radiology. 2018;73(8):700-714. DOI: 10.1016/j.crad.2017.10.021.

17. Safarpour Lima Z, Ebadi M, Amjad G, et al. Application of imaging technologies in breast cancer detection: a review article. Macedonian Journal of Medical Sciences. 2019;7(5):838-848. DOI: 10.3889/oamjms.2019.171

18. Tan KP, Azlan ZM, Choo MY, et al. The comparative accuracy of ultrasound and mammography in the detection of breast cancer. Med $J$ Malaysia. 2014;69(2):79-85. 